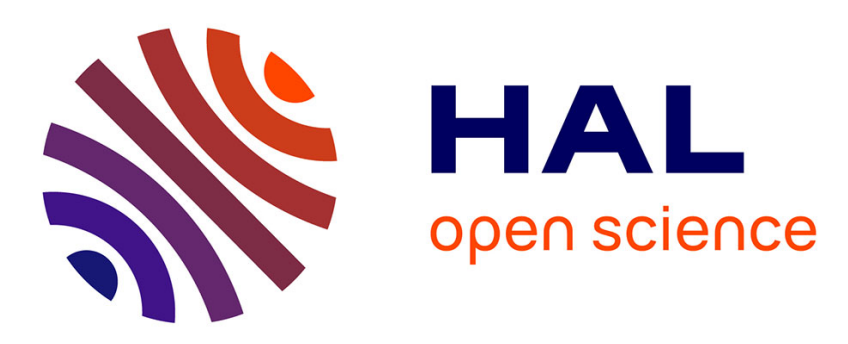

\title{
Long-Lived States in Hyperpolarized Deuterated Methyl Groups Reveal Weak Binding of Small Molecules to Proteins
}

Thomas Kress, Astrid Walrant, Geoffrey Bodenhausen, Dennis Kurzbach

\section{- To cite this version:}

Thomas Kress, Astrid Walrant, Geoffrey Bodenhausen, Dennis Kurzbach. Long-Lived States in Hyperpolarized Deuterated Methyl Groups Reveal Weak Binding of Small Molecules to Proteins. Journal of Physical Chemistry Letters, 2019, 10 (7), pp.1523-1529. 10.1021/acs.jpclett.9b00149 • hal-02093315

\section{HAL Id: hal-02093315 https://hal.sorbonne-universite.fr/hal-02093315}

Submitted on 8 Apr 2019

HAL is a multi-disciplinary open access archive for the deposit and dissemination of scientific research documents, whether they are published or not. The documents may come from teaching and research institutions in France or abroad, or from public or private research centers.
L'archive ouverte pluridisciplinaire HAL, est destinée au dépôt et à la diffusion de documents scientifiques de niveau recherche, publiés ou non, émanant des établissements d'enseignement et de recherche français ou étrangers, des laboratoires publics ou privés. 


\title{
, Long-Lived States in Hyperpolarized Deuterated Methyl Groups ${ }_{2}$ Reveal Weak Binding of Small Molecules to Proteins
}

\author{
${ }_{3}$ Thomas Kress, ${ }^{\dagger, \perp}$ Astrid Walrant, ${ }^{\ddagger}$ Geoffrey Bodenhausen, ${ }^{*}{ }^{\dagger}$ and Dennis Kurzbach ${ }^{*}, \perp_{\odot}$ \\ $4{ }^{\dagger}$ Laboratoire des Biomolécules, LBM, Département de Chimie, École Normale Supérieure, PSL University, Sorbonne Université, \\ 5 CNRS, 75005 Paris, France \\ $6{ }^{*}$ Laboratoire des Biomolécules, LBM, École Normale Supérieure, Sorbonne Université, École Normale Supérieure, PSL University, \\ 7 CNRS, 75005 Paris, France \\ ${ }_{8}^{\perp}$ Faculty of Chemistry, Institute of Biological Chemistry, University of Vienna, Währinger Strasse 38, 1090 Vienna, Austria
}

ABSTRACT: We introduce a method for the detection of weak interactions of small molecules such as metabolites or medicaments that contain deuterated methyl groups with proteins in solution. The technique relies on long-lived imbalances of spin state populations, which are generated by dissolution dynamic nuclear polarization (D-DNP) and feature lifetimes that depend on the frequency of internal rotation of deuterated methyl groups. We demonstrate the technique for interactions between deuterated dimethyl sulfoxide (DMSO- $d_{6}$ ) and bovine serum albumin (BSA) or trypsin, where the methyl group rotation is slowed down upon protein binding, which causes a marked reduction in the lifetime of the population imbalances.

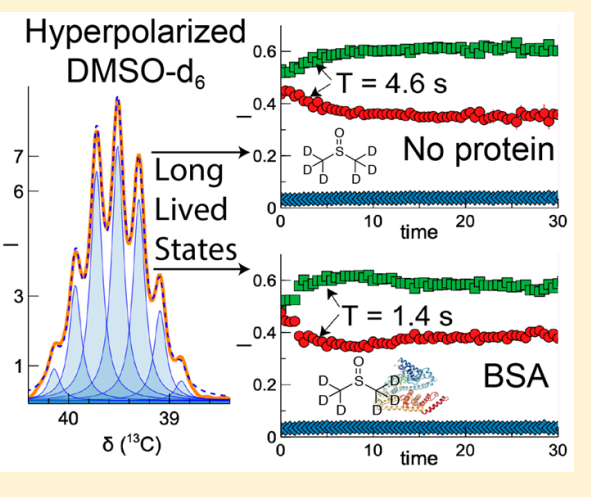

${ }_{21}^{20} \mathrm{D}$ euterated drugs have gained ample attention in the recent past as deuteration of selected moieties in 22 established medicaments can lead to improved pharmacoki23 netic properties. ${ }^{1}$ Because $\mathrm{C}-\mathrm{D}$ bonds $\left(\mathrm{D}={ }^{2} \mathrm{H}\right)$ are more 24 stable than $\mathrm{C}-\mathrm{H}$ bonds with respect to enzymatic degradation, 25 deuterated drugs feature a prolonged half-life time in the 26 body, $^{2}$ which allows one to reduce their dosage and attenuate 27 side effects. ${ }^{3,4}$ From an analytical viewpoint, deuteration of 28 drugs offers novel possibilities for nuclear magnetic resonance 29 (NMR) spectroscopy. NMR is a key method to investigate 30 interactions of both drugs and target proteins. Complementary 31 to methods such as isothermal titration calorimetry (ITC) and 32 surface plasmon resonance (SPR) that provide global 33 thermodynamic and kinetic parameters about drug/target 34 systems, solution-state NMR can localize drug binding sites 35 and characterize their dynamics at atomic resolution. In 36 addition, the affinity of drugs or their metabolites for 37 macromolecules such as proteins ${ }^{5}$ can be studied by a wealth 38 of NMR techniques that exploit, for example, contrasts in $T_{2}$ or $39 T_{1 \rho}$ as well as relaxation rates of long-lived states or long-lived 40 coherences upon binding. ${ }^{6,7}$ Alternatively, differences in signal 41 intensities can be observed upon selective saturation of one of 42 the binding partners, known as saturation transfer difference 43 (STD) spectroscopy, ${ }^{8,9}$ or of the solvent, known as water44 LOGSY. ${ }^{10,11}$ In the past years, dissolution dynamic nuclear 45 polarization (D-DNP) has also become increasingly popular as 46 a tool for drug screening and ligand binding studies. For 47 example, Lerche et al. have demonstrated how to use carbon4813 signal losses to identify the binding of small ligands to macromolecular hosts; ${ }^{12}$ Hilty and co-workers have shown 49 how to employ relaxation dispersion techniques in combina- 50 tion with ${ }^{13} \mathrm{C}$ and ${ }^{19} \mathrm{~F}$ detection, ${ }^{13,14}$ while Wilson and co- 51 workers used changes in longitudinal ${ }^{13} \mathrm{C}$ relaxation upon 52 ligand binding in magnetic resonance imaging. ${ }^{15}$

To expand NMR and D-DNP to deuterated drugs and 54 metabolites, we enlarge the NMR toolbox by monitoring 55 effects on long-lived nuclear spin states (LLS) in deuterated 56 methyl groups $\left(\mathrm{CD}_{3}\right)$. The relaxation rates $R_{\mathrm{LLS}}=1 / T_{\mathrm{LLS}}$ of 57 these LLS are sensitive to the frequency of internal $\mathrm{CD}_{3} 58$ rotation, characterized by the frequency, thus enabling one to 59 identify interactions between target proteins and drugs or 60 metabolites containing deuterated methyl groups. LLS 61 involving two or more deuterons have been described as 62 imbalances between populations of nuclear spin states of 63 different symmetry. ${ }^{16}$ The flow of populations between 64 different symmetry manifolds is forbidden to first order, so 65 that population imbalances have a prolonged lifetime 66 compared to spin-lattice relaxation times.

Different kinds of LLS have been reported in $\mathrm{CH}_{2}$ and $\mathrm{CH}_{3} 68$ groups by Dumez, Levitt, Jannin, and co-workers ${ }^{17-19}$ and in 69 $\mathrm{CD}_{2}$ and $\mathrm{CD}_{3}$ groups by Kurzbach et al. ${ }^{20}$ and by Jhaharia et 70 $\mathrm{al}^{21}$ In deuterated methyl groups, LLS return to equilibrium 71 populations with rates that depend on (i) the correlation time 72 
$73 \tau_{\mathrm{c}}$ of the overall rotational diffusion of the molecule (here 74 assumed to be isotropic), (ii) the internal rotation of the 75 methyl group around its three-fold symmetry axis with a 76 correlation time $\tau_{\mathrm{c}} \ll \tau_{\mathrm{r}}$. Both $\tau_{\mathrm{c}}$ and $\tau_{\mathrm{r}}$ can be affected by the 77 binding of a molecule comprising a deuterated $\mathrm{CD}_{3}$ group to a 78 target protein, hence revealing their mutual interaction.

79 To produce the LLS that serve here as sensors for host80 guest interactions, we applied the $\mathrm{D}-\mathrm{DNP}^{22}$ technique to 81 deuterated dimethyl sulfoxide (DMSO- $d_{6}$ ) with carbon-13 in 82 natural abundance, which we shall use as a model for drugs or 83 metabolites containing deuterated methyl groups.

84 D-DNP aims at boosting NMR signal intensities by 85 transferring the magnetization of unpaired electrons of 86 paramagnetic agents (PAs) such as $1-\lambda^{1}$-oxidanyl-2,2,6,687 tetramethylpiperidin-4-ol (TEMPOL) to the nuclear spins of 88 a molecule of interest. Our D-DNP experiments proceeded in 89 three steps: (i) Partial saturation of the electron spin resonance 90 spectrum of samples containing $50 \mathrm{mM}$ TEMPOL using 91 microwave irradiation $\left(\nu_{\mu \mathrm{w}}=187.9 \mathrm{GHz}, B_{0, \mathrm{DNP}}=6.7 \mathrm{~T}\right)$, 92 which results in direct positive hyperpolarization of both ${ }^{13} \mathrm{C}$ 93 and ${ }^{2} \mathrm{H}$ nuclei. (The optimal microwave frequencies for these 94 nuclei do not differ significantly under our experimental 95 conditions. $\left.{ }^{23}\right)$ Nuclear hyperpolarizations $P_{\mathrm{I}}\left({ }^{13} \mathrm{C}\right)>15 \%$ and $96 P_{\mathrm{I}}\left({ }^{2} \mathrm{H}\right)>15 \%$ can be achieved at cryogenic temperatures of $97 T_{\mathrm{DNP}}=1.2 \mathrm{~K}$. The deuterium hyperpolarization reaches a 98 plateau after saturating the EPR transitions of TEMPOL by 99 microwave irradiation for ca. $1 \mathrm{~h}$ (see Figure S6, Supporting 100 Information) with a characteristic time constant of ca. $18 \mathrm{~min}$. 101 (ii) After the polarization has built up, the frozen hyper102 polarized sample is dissolved with $5 \mathrm{~mL}$ of overheated $\mathrm{D}_{2} \mathrm{O}$ $103\left(T_{\mathrm{D} 2 \mathrm{O}}=453 \mathrm{~K}\right.$ at $\left.P_{\mathrm{D} 2 \mathrm{O}}=1.05 \mathrm{MPa}\right)$ and pushed within $3 \mathrm{~s}$ 104 through a magnetic tunnel $\left(B_{0, \text { tunnel }}=0.9 \mathrm{~T}\right)$ to a solution-state 105 NMR spectrometer, where a fraction of ca. $1 \mathrm{~mL}$ of the bolus is 106 injected into $1 \mathrm{~mL}$ of a buffer solution with or without target 107 proteins (Figure 1). (iii) For all experiments reported here, ${ }^{13} \mathrm{C}$ 108 NMR spectra of hyperpolarized DMSO- $d_{6}$ are acquired at 109 intervals of $0.5 \mathrm{~s}$ by applying $15^{\circ}$ detection pulses (see Figure $110 \mathrm{~S} 1$ in the SI). Spectra are acquired up to $80 \mathrm{~s}$ after injection, 111 when the signals become too weak for detection.

112 The LLS were monitored indirectly via ${ }^{13} \mathrm{C}$ NMR of the 113 multiplets of the $\mathrm{CD}_{3}$ groups. In thermal equilibrium, the 114 spectrum contains seven lines (see Figure 2) that feature a 115 trinomial intensity distribution $1: 3: 6: 7: 6: 3: 1$, which results 116 from couplings ${ }^{1} J\left({ }^{13} \mathrm{C},{ }^{2} \mathrm{H}\right)=21.2 \mathrm{~Hz}$ to three magnetically 117 equivalent deuterium spins with spin $I=1$. After a DNP 118 buildup time of $>1 \mathrm{~h}$ without cross-polarization, the ${ }^{13} \mathrm{C}$ 119 multiplets benefit from ca. 10 000-fold signal enhancements 120 with respect to their amplitudes in thermal equilibrium 121 (typically detected by averaging 1024 scans over a period of $12296 \mathrm{~h}$ ). The time dependence of the seven line intensities after 123 injection allows one to determine the lifetime of the LLS. 124 Figure 2 shows a hyperpolarized multiplet $3 \mathrm{~s}$ after injection 125 (Figure 2a) and a partially relaxed spectrum $60 \mathrm{~s}$ after injection 126 (Figure 2b). The population imbalance is evidenced, inter alia, 127 through increased relative intensities of lines $L_{1}$ and $L_{7}$ and a 128 reduced relative intensity of $L_{4}$. Note that the multiplets are 129 asymmetric about their centers (e.g., line $L_{1}$ is stronger than $L_{7}$ 130 etc.), hinting at the presence of $\mathrm{C}_{z} \mathrm{D}_{z}$ and $\mathrm{C}_{z} \mathrm{D}_{z} \mathrm{D}_{z}{ }^{\prime} \mathrm{D}_{z}{ }^{\prime \prime}$ order 131 (see Figure S4 in the SI).

132 Monitoring of LLS. The averaged ("lumped") populations ${ }^{12}$ $133 P_{\mathrm{A}}, P_{\mathrm{B}}$, and $P_{\mathrm{E}}$ of the three manifolds of symmetry $\mathrm{A}, \mathrm{B}$, and $\mathrm{E}$ $134=\mathrm{E}_{1} \oplus \mathrm{E}_{2}$ can be monitored as a function of time after 135 injection. The lifetime $T_{\mathrm{LLS}}\left({ }^{2} \mathrm{H}\right)$ was up to 6 times longer than

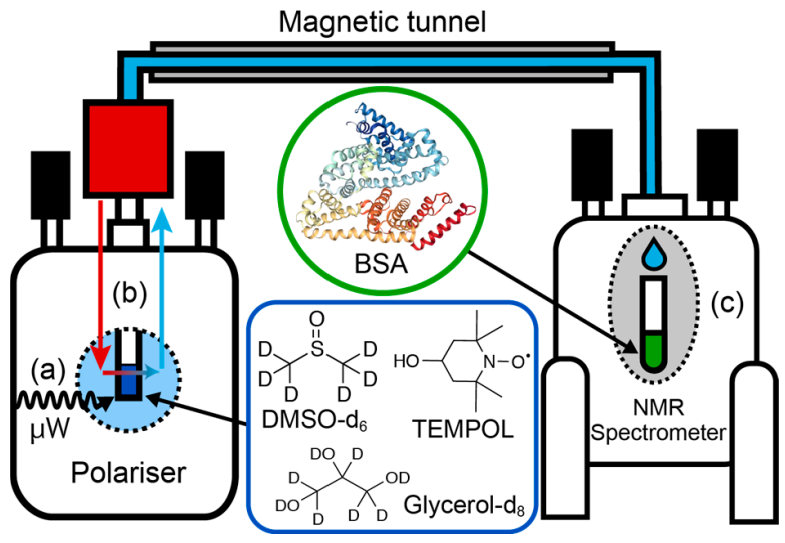

Figure 1. Hyperpolarized NMR can be exploited for the detection of LLS in ligands, drugs, or metabolites comprising deuterated $\mathrm{CD}_{3}$ groups. A frozen solution (blue) of a ligand such as DMSO- $d_{6}$ containing $50 \mathrm{mM}$ of the radical TEMPOL is hyperpolarized by microwave irradiation (here at $187.9 \mathrm{GHz}$ ) in a dedicated DNP apparatus that operates at a temperature of $1.2 \mathrm{~K}$ and a magnetic field of $6.7 \mathrm{~T}$. The sample is then dissolved with a burst of $5 \mathrm{~mL}$ of superheated $\mathrm{D}_{2} \mathrm{O}$ at 10.5 bar and $180^{\circ} \mathrm{C}$ (red box) and transferred within $3 \mathrm{~s}$ to an NMR spectrometer operating at $9.4 \mathrm{~T}(400 \mathrm{MHz}$ for ${ }^{1} \mathrm{H}$ ) where a fraction is injected into an NMR tube that contains a buffered protein solution (green). Finally, a series of ${ }^{13} \mathrm{C}$ NMR spectra of the ligand DMSO- $d_{6}$ are acquired at regular intervals after injection.

$T_{1}\left({ }^{2} \mathrm{H}\right)$ in DMSO- $d_{6}$ under our experimental conditions. 136 Figure $2 \mathrm{c}$ shows the energy levels in a $\mathrm{D}_{3}$ spin system with 137 their assignment to different symmetry manifolds. The 138 following normalized linear combinations of line intensities 139 allow one to determine the lumped normalized populations ${ }^{16} 140$

$$
\begin{aligned}
& P_{\mathrm{A}}=5\left(L_{1}+L_{7}\right) / L_{\text {tot }} \\
& P_{\mathrm{B}}=\left[L_{4}-\frac{1}{2}\left(L_{3}+L_{5}\right)\right] / L_{\text {tot }} \\
& P_{\mathrm{E}}=\left[2\left(L_{3}+L_{5}\right)-4 L_{1}-4 L_{7}\right] / L_{\text {tot }} \\
& L_{\text {tot }}=\sum_{i} L_{i}
\end{aligned}
$$

Figure 3 depicts the evolution of the lumped normalized $142 \mathrm{f} 3$ populations $P_{\mathrm{A}}, P_{\mathrm{B}}$, and $P_{\mathrm{E}}$ after injection into the NMR 143 spectrometer. They tend exponentially toward their thermal 144 equilibrium distribution where all 27 states have approximately 145 the same populations $\left(P_{\mathrm{A}}^{\mathrm{eq}}=10 / 27 \simeq 0.37 ; P_{\mathrm{E}}^{\mathrm{eq}}=16 / 27 \simeq 146\right.$ $\left.0.59 ; P_{\mathrm{B}}^{\mathrm{eq}}=1 / 27 \simeq 0.037\right)$. Evidently, the LLS corresponds to 147 an overpopulation $P_{\mathrm{A}} / P_{\mathrm{A}}^{\text {eq }}>1$ and a deficiency $P_{\mathrm{E}} / P_{\mathrm{E}}^{\text {eq }}<1$, i.e., 148 to a positive $\mathrm{A} / \mathrm{E}$ spin-state imbalance with respect to thermal 149 equilibrium, where $P_{\mathrm{A}}^{\mathrm{eq}} / P_{\mathrm{E}}^{\mathrm{eq}}=10 / 16=0.625$. Once turbulences 150 after injection into the NMR spectrometer have died out (see 151 the SI), we find initial polarizations up to $P_{\mathrm{A}} \approx 0.46$ or $P_{\mathrm{A}} / P_{\mathrm{A}}^{\text {eq }} 152$ $\approx 1.24$ and $P_{\mathrm{E}} \approx 0.51$ or $P_{\mathrm{E}} / P_{\mathrm{E}}^{\mathrm{eq}} \approx 0.86$ and a ratio $P_{\mathrm{A}} / P_{\mathrm{E}} \approx 0.9 .153$ By analogy to earlier work, ${ }^{24}$ the polarization of the long-lived 154 A/E imbalance can be defined as a ratio

$$
P_{\mathrm{LLS}}\left({ }^{2} \mathrm{H}\right)=\left(\frac{P_{\mathrm{A}}}{P_{\mathrm{A}}^{\text {eq }}}-\frac{P_{\mathrm{E}}}{P_{\mathrm{E}}^{\text {eq }}}\right) /\left(\frac{P_{\mathrm{A}}}{P_{\mathrm{A}}^{\text {eq }}}+\frac{P_{\mathrm{E}}}{P_{\mathrm{E}}^{\text {eq }}}\right)
$$

which in the case at hand (Figure 3 ) is estimated to be ca. $18 \% .157$ The decay time constants of the imbalance $P_{\mathrm{LLS}}\left({ }^{2} \mathrm{H}\right)$ can be as 158 long as $T_{\mathrm{LLS}}\left({ }^{2} \mathrm{H}\right)=4.3 \mathrm{~s}$ in protein-free, buffered solutions 159 

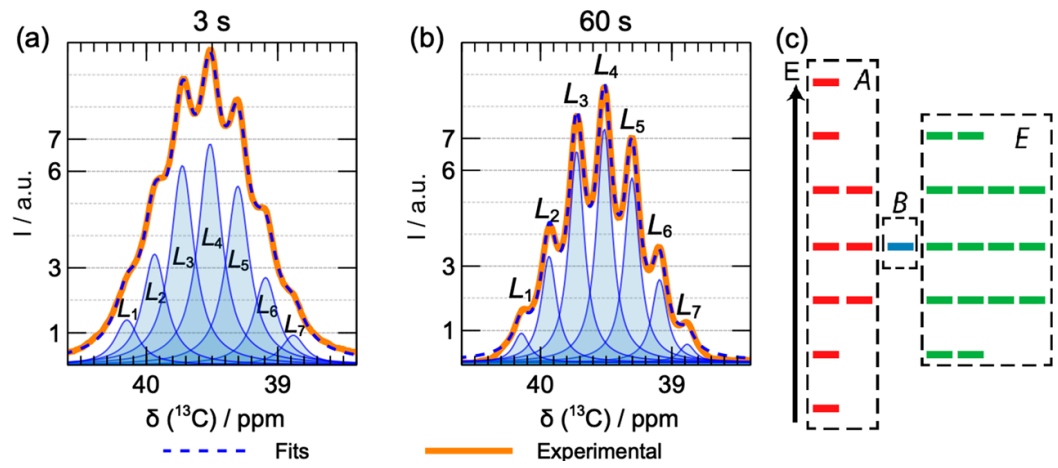

Figure 2. Typical ${ }^{13} \mathrm{C}$ multiplets of the ${ }^{13} \mathrm{CD}_{3}$ groups in DMSO- $d_{6}$ (deuteration $>99.9 \%$ to reduce signals of partly protonated methyl groups) with ${ }^{13} \mathrm{C}$ in natural abundance obtained (a) 3 and (b) $60 \mathrm{~s}$ after injection into a buffer solution without any proteins. The LLS lead to deviations of the septet from the trinomial high-temperature intensity distribution 1:3:6:7:6:3:1, in particular, to attenuation of the inner line intensities $L_{3}, L_{4}, L_{5}$, and $L_{6}$ compared to the outer lines $L_{1}$ and $L_{7}$. The signals of the seven components are shown in light blue; their sums are represented by dashed blue lines. The fit with the experimental multiplets (orange lines) is satisfactory. (c) Energy-level diagram of a $\mathrm{D}_{3}$ spin system with 27 states belonging to three different irreducible representations of the $C_{3 v}$ point group $\mathrm{A}, \mathrm{B}$, and $\mathrm{E}$. By D-DNP with microwave irradiation of the electron spin transitions of TEMPOL radicals at $1.2 \mathrm{~K}$, states of symmetry A can be overpopulated, while states of symmetry B and E are partly depleted, thus boosting lines $L_{1}$ and $L_{7}$ in the multiplet. ${ }^{16}$ The number of spin states is increased to 54 when the ${ }^{13} \mathrm{C}$ nucleus is taken into account.

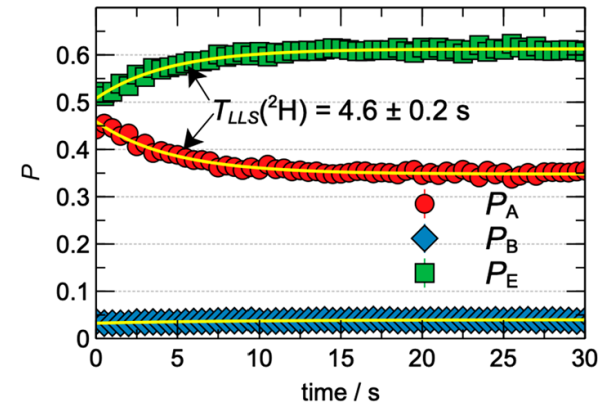

Figure 3. Evolution of the normalized lumped populations $P_{\mathrm{A}}, P_{\mathrm{B}}$, and $P_{\mathrm{E}}$ determined from seven experimental line intensities $L_{i}$ via eq 1 as a function of time after turbulences have died out (see the SI; $t=0 \mathrm{~s}$ in the figure corresponds to $2 \mathrm{~s}$ after injection). The fitted time constant of the decay of the A/E imbalance is $T_{\text {LLS }}\left({ }^{2} \mathrm{H}\right)=4.6 \pm 0.2 \mathrm{~s}$. The vertical scale was calibrated by normalizing the total polarization to unity $\left(P_{\mathrm{A}}+P_{\mathrm{B}}+P_{\mathrm{E}}=1\right)$.

160 (Figure 3), which is ca. 6 times longer than $T_{1}\left({ }^{2} \mathrm{H}\right)=0.7 \mathrm{~s}$. 161 Note that normalized populations are not affected by 162 longitudinal ${ }^{13} \mathrm{C}$ relaxation.

163 The curves in Figure 3 can be understood with the help of 164 simulations with SpinDynamica ${ }^{25}$ (see the SI). Figure 4 shows 165 that the excess population of the A states flows toward the $\mathrm{E}$ 166 states and, to a lesser extent, toward the $\mathrm{B}$ state, until the 167 equilibrium is reached. The rate $R_{\mathrm{LLS}}\left({ }^{2} \mathrm{H}\right)=1 / T_{\mathrm{LLS}}\left({ }^{2} \mathrm{H}\right)$ of the 168 flow between $\mathrm{A}$ and $\mathrm{E}$ states (which is actually the average of 169 two different rates; see the SI) depends (i) on the correlation 170 time $\tau_{c}$ of overall tumbling of the molecule that carries the 171 methyl group (or of the transient complex that this molecule 172 may form with a macromolecular partner), (ii) on the 173 correlation time $\tau_{\mathrm{r}}$ of the internal methyl group rotation, and 174 (iii) on the quadrupolar coupling constant $\left\langle\omega_{\mathrm{Q}}\right\rangle$ that is 175 averaged by rapid internal rotation ${ }^{16}$

$$
\begin{aligned}
& R_{\mathrm{LLS}}=\frac{15}{4} \frac{\tau_{\mathrm{c}} \tau_{\mathrm{r}}\left(2 \tau_{\mathrm{c}}+\tau_{\mathrm{r}}\right)\left\langle\omega_{\mathrm{Q}}\right\rangle^{2}}{\left(\tau_{\mathrm{c}}+\tau_{\mathrm{r}}\right)\left(4 \tau_{\mathrm{c}}+\tau_{\mathrm{r}}\right)} \\
& \sim \frac{15}{8} \tau_{\mathrm{r}}\left\langle\omega_{\mathrm{Q}}\right\rangle^{2} \quad \text { for } \tau_{\mathrm{r}} \ll \tau_{\mathrm{c}}
\end{aligned}
$$
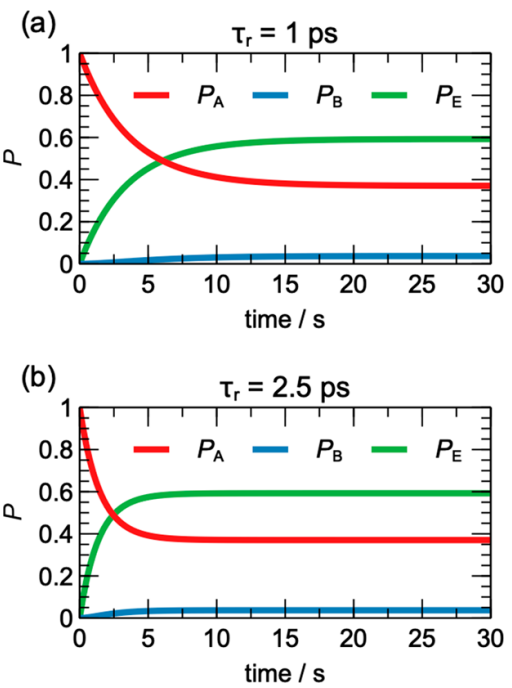

Figure 4. Simulated time traces of the averaged (lumped) populations $P_{\mathrm{A}}$ (red), $P_{\mathrm{E}}$ (green), and $P_{\mathrm{B}}$ (blue) in a $\mathrm{D}_{3}$ spin system for $\left\langle\omega_{\mathrm{Q}}\right\rangle /(2 \pi)=67 \mathrm{kHz}, \tau_{\mathrm{c}}=100 \mathrm{ps}$, and (a) $\tau_{\mathrm{r}}=1 \mathrm{ps}$ and $(\mathrm{b}) \tau_{\mathrm{r}}=2.5$ ps, starting from a hypothetical "perfect" initial imbalance $P_{\mathrm{A}}=1$; $P_{\mathrm{B}}=P_{\mathrm{E}}=0$. In our experiments, the initial conditions are $P_{\mathrm{A}}<1$, $P_{\mathrm{B}}>0$, and $P_{\mathrm{E}}>0$. The rates $R_{\mathrm{LLS}}$ are 0.26 and $0.64 \mathrm{~s}^{-1}$ in (a) and (b), respectively. Note that the values of $\tau_{\mathrm{c}}$ and $\tau_{\mathrm{r}}$ used for the simulations serve only for demonstration and should be considered with caution. The fitting of complex relaxation equations to experimental time traces is largely underdetermined for the case at hand (see ref 26).

The simplification of eq 4 is allowed assuming that $0.1<\tau_{\mathrm{c}}<178$ $1 \mathrm{~ns}$ for DMSO- $d_{6}$ in complexes with macromolecular partners, 179 while it is $0.1<\tau_{\mathrm{r}}<10 \mathrm{ps}$ for methyl group rotation in DMSO- 180 $d_{6}$, so that the inequality $\tau_{\mathrm{r}} \ll \tau_{\mathrm{c}}$ is fulfilled by at least 1 order 181 of magnitude. Thus, the rate $R_{\mathrm{LLS}}$ in eq 4 is directly 182 proportional to the correlation time of the internal methyl 183 group rotation. For example, if $\tau_{\mathrm{c}}=0.1 \mathrm{~ns}$ and $\left\langle\omega_{\mathrm{Q}}\right\rangle /(2 \pi)=67184$ $\mathrm{kHz}$, an increase in $\tau_{\mathrm{R}}$ from 1 to 2.5 ps would entail a drop in 185 $T_{\text {LLS }}\left({ }^{2} \mathrm{H}\right)$ from 3.8 to $1.6 \mathrm{~s}$ that should be readily observable in 186 our experiments. LLS relaxation times $1<T_{\text {LLS }}\left({ }^{2} \mathrm{H}\right)<30 \mathrm{~s}$ lie 187 

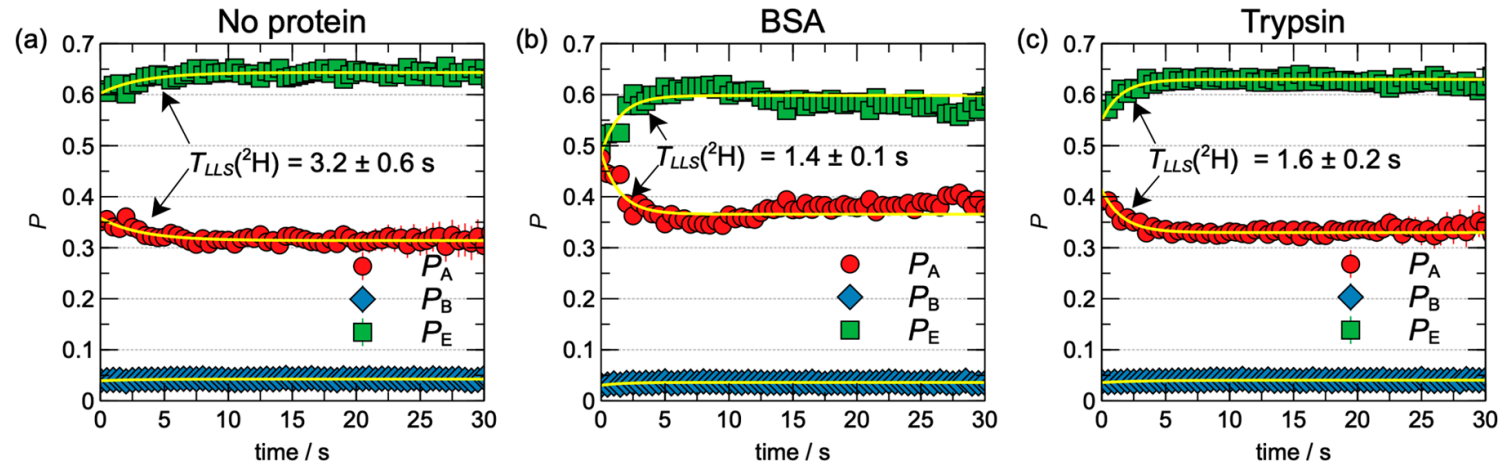

Figure 5. Decay of the lumped populations $P_{\mathrm{A}}$ (red), $P_{\mathrm{E}}$ (green), and $P_{\mathrm{B}}$ (blue) of the three symmetry manifolds upon injection into (a) neat buffer, (b) BSA, and (c) trypsin solutions. The return of the populations to equilibrium is the slowest, i.e., the lifetime $T_{\mathrm{LLS}}\left({ }^{2} \mathrm{H}\right)$ is the longest, in the absence of any protein and shorter in the presence of BSA than in solutions containing trypsin, indicating a stronger affinity and a more restrained internal methyl group rotation in the former case. The vertical scale was calibrated by normalizing the total polarization to unity $\left(P_{\mathrm{A}}+P_{\mathrm{B}}+P_{\mathrm{E}}=1\right)$. The TEMPOL concentrations after injection were comparable in all three experiments, as evidenced by comparable $T_{1}\left({ }^{13} \mathrm{C}\right),(\mathrm{a}) 16.6 \pm 0.1,(\mathrm{~b})$ $16.4 \pm 0.1$, and (c) $14.7 \pm 0.1 \mathrm{~s}$, so that it is legitimate to compare the three experiments (see the main text).

188 in an appropriate range for detection by ${ }^{13} \mathrm{C} \mathrm{NMR}$, bearing in 189 mind that very fast processes with $T_{\mathrm{LLS}}\left({ }^{2} \mathrm{H}\right)<1 \mathrm{~s}$ cannot be 190 detected by current D-DNP technology due to the relatively 191 slow transfer from the polarizer to the NMR system, and very 192 slow processes with $T_{\text {LLS }}\left({ }^{2} \mathrm{H}\right)>30 \mathrm{~s}$ would be masked by 193 longitudinal ${ }^{13} \mathrm{C}$ relaxation, with lifetimes that are typically on 194 the order of $T_{1}\left({ }^{13} \mathrm{C}\right)=30 \mathrm{~s}$ in DMSO- $d_{6}$ in the absence of 195 radicals.

196 Note that, provided $\tau_{\mathrm{c}} \gg \tau_{\mathrm{r}}$, longitudinal relaxation times ${ }_{197} T_{1}\left({ }^{2} \mathrm{H}\right)$ and $T_{1}\left({ }^{13} \mathrm{C}\right)$ in methyl groups primarily depend on $\tau_{\mathrm{c}}$ 198 rather than on $\tau_{\mathrm{r}}$, while the opposite situation arises for $199 T_{\text {LLS }}\left({ }^{2} \mathrm{H}\right)$ which depends mostly on $\tau_{\mathrm{r}}$ and barely on $\tau_{\mathrm{c}}$. Hence $200 T_{\text {LLS }}\left({ }^{2} \mathrm{H}\right)$ can report on small variations of $\tau_{\mathrm{r}}$ caused by weak 201 interactions with macromolecular partners that lead to some 202 steric hindrance to rotation. Furthermore, it follows from eq 4 203 that $T_{\text {LLS }}\left({ }^{2} \mathrm{H}\right)$ is largely independent of the mass of the 204 complex formed with the macromolecular partner but depends 205 on steric constraints that slow down the internal methyl group 206 rotation.

207 Because quadrupolar relaxation is not very effective in 208 diminishing an $\mathrm{A} / \mathrm{E}$ imbalance, as evidenced by the long 209 lifetimes $T_{\text {LLS }}\left({ }^{2} \mathrm{H}\right)$, one may wonder if other relaxation 210 mechanisms might become important. Dipolar ${ }^{2} \mathrm{H}$-relaxation 211 is subject to similar symmetry constraints as quadrupolar 212 relaxation, i.e., a flow of polarization between spin manifolds 213 that belong to different irreducible representations within the $214 C_{3 v}$ point group through dipolar relaxation is also forbidden by 215 first order, as discussed by Dumez et al. ${ }^{26}$ and Ivanov et al. ${ }^{16}$ 216 However, dipolar couplings between deuterons are over 1 217 order of magnitude weaker than quadrupolar couplings. 218 Numerical simulations show that the effect of dipolar 219 relaxation on an $\mathrm{A} / \mathrm{E}$ imbalance is negligible compared to 220 quadrupolar relaxation (see the SI). However, paramagnetic 221 relaxation due to radicals such as TEMPOL significantly affects 222 both $T_{\text {LLS }}\left({ }^{2} \mathrm{H}\right)$ and $T\left({ }^{13} \mathrm{C}\right)$, as shown in Figure 6.

223 Monitoring Ligand-Protein Interactions. The dependence of $224 T_{\mathrm{LLS}}\left({ }^{2} \mathrm{H}\right)$ on $\tau_{\mathrm{r}}$ described by eq 4 can be put to use to identify 225 ligand-protein interactions that are likely to perturb the 226 methyl group rotation. This may be of interest for small 227 molecules or ligands that are being considered as potential 228 drugs. We compared interactions of DMSO- $d_{6}$ with bovine 229 serum albumin (BSA) and trypsin from porcine pancreas. BSA 230 is a transporter protein with binding pockets that can accommodate small ligands such as DMSO, as evidenced by 231 the effect of DMSO on PCR amplification. ${ }^{27}$ In contrast, 232 DMSO- $d_{6}$ is expected to interact less efficiently with trypsin, 233 which is not a transporter protein. Hence, deuterated methyl 234 groups in DMSO- $d_{6}$ are expected to be less perturbed by 235 trypsin than by BSA, and the LLS are expected to live longer in 236 the former case.

237

All $T_{\text {LLS }}\left({ }^{2} \mathrm{H}\right)$ experiments were carried out in $10 \mathrm{mM} 238$ phosphate buffer saline (PBS) adjusted to $\mathrm{pH}$ 7.4. The final 239 protein concentrations were $225 \pm 45 \mu \mathrm{M}$, and the final ligand 240 concentrations were $350 \pm 150 \mathrm{mM}$ depending on the 241 injection (see the SI). Figure 5 shows that the lifetimes can be $242 \mathrm{fs}$ ranked in the sequence $T_{\mathrm{LLS}}($ protein-free $)=3.2 \pm 0.6 \mathrm{~s}>243$ $T_{\text {LLS }}($ trypsin $)=1.6 \pm 0.2 \mathrm{~s}>T_{\text {LLS }}($ BSA $)=1.4 \pm 0.1 \mathrm{~s}$. As 244 expected, the decay is faster with BSA than that with trypsin. 245 When considering the fast equilibrium between free and bound 246 DMSO and the interactions on the surface or in the binding 247 pockets, the methyl group rotation appears to be more 248 constrained in the DMSO/BSA complex than that in the 249 $\mathrm{DMSO} /$ trypsin case.

250

The ligand-protein interactions were studied under similar 251 experimental conditions by ITC: flat ITC profiles (see Figure 252 $\mathrm{S} 2$ in the SI) indicate that the interactions between DMSO- $d_{6} 253$ and BSA are nonspecific, with an interaction heat $\Delta H=+142.6254$ $\pm 14.6 \mathrm{~J} / \mathrm{mol}$ at $298 \mathrm{~K}$, while interactions between DMSO- $d_{6} 255$ and trypsin yield $\Delta H=+119.3 \pm 6.7 \mathrm{~J} / \mathrm{mol}$. This points 256 toward slightly stronger nonspecific binding of DMSO- $d_{6}$ to 257 BSA than to trypsin, presumably because BSA offers 258 amphiphilic binding pockets. $^{28}$

259

Reproducibility. Because of shortcomings of current in- 260 strumentation, D-DNP experiments can be subject to 261 significant uncertainties, in particular, when the volume of 262 the injected solutions and the final concentrations of the 263 hyperpolarized molecules and radicals are not perfectly 264 controlled. In most D-DNP studies that have been carried 265 out so far, such variations had little effect on the outcome of 266 the experiments. However, in comparisons such as those 267 illustrated in Figure 5, one must pay attention to various 268 details. First, the reproducibility of the dissolution process is 269 necessarily limited because the frozen glassy sample that was 270 hyperpolarized at $1.2 \mathrm{~K}$ must be dissolved by a burst of 271 superheated solvent. The efficiency of this process depends (i) 272 on the speed of the dissolution (which determines the fraction 273 
274 of surviving hyperpolarization), which in turn depends on the 275 volume and morphology of the frozen sample (e.g., a solid 276 block, small granules, etc.) and (ii) on the fraction of the heat 277 capacity of the superheated solvent that is wasted upon 278 warming up the probe and cryostat. As a consequence of these 279 factors, the bolus of the hyperpolarized liquid that travels from 280 the polarizer to the NMR spectrometer before injection may 281 feature a varying concentration gradient, which affects the final 282 concentration if only $20 \%$ (here, the leading $1 \mathrm{~mL}$ fraction out 283 of $5 \mathrm{~mL}$ ) of the bolus is injected (see the SI for details). Such 284 factors influence the final ligand and TEMPOL concentrations, 285 which, if not appropriately controlled, can lead to significant 286 variations in the observed relaxation rates.

287 Figure 6 shows empirical correlations of $T_{\text {LLS }}\left({ }^{2} \mathrm{H}\right)$ and $288 T_{1}\left({ }^{13} \mathrm{C}\right)$ for $4-6$ independent repetitions of experiments on

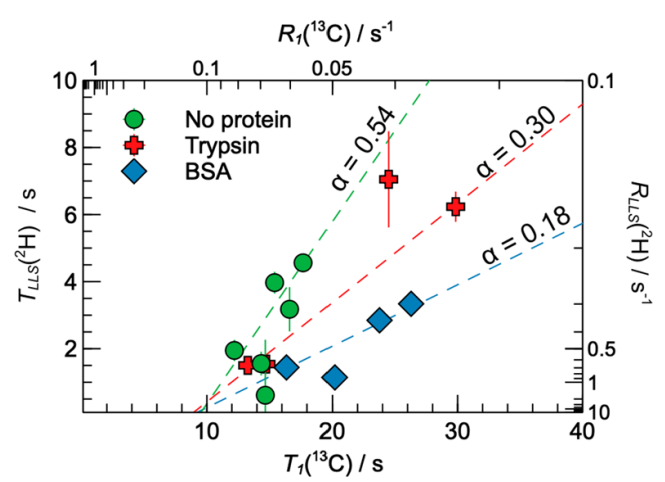

Figure 6. Correlations of lifetimes $T_{\mathrm{LLS}}\left({ }^{2} \mathrm{H}\right)$ vs $T_{1}\left({ }^{13} \mathrm{C}\right)$ in DMSO- $d_{6}$ measured in six independent D-DNP experiments without target protein (green dots), four experiments in the presence of trypsin (red crosses), and four experiments in the presence of BSA (blue diamonds). Variations in TEMPOL concentrations upon injection (see main text) affect both $T_{\mathrm{LLS}}\left({ }^{2} \mathrm{H}\right)$ and $T_{1}\left({ }^{13} \mathrm{C}\right)$ by paramagnetic relaxation enhancement. The dashed lines indicate the slopes $\alpha=$ $\Delta T_{\mathrm{LLS}}\left({ }^{2} \mathrm{H}\right) / \Delta T_{1}\left({ }^{13} \mathrm{C}\right.$ ) for the three different cases (no protein, trypsin, or BSA). Variations of the longitudinal relaxation times $T_{1}\left({ }^{13} \mathrm{C}\right)$ reflect variations of the TEMPOL concentration, while deuterium relaxation $T_{1}\left({ }^{2} \mathrm{H}\right)$ is not significantly affected (see the SI).

289 three different samples (altogether 14 independent runs): 290 protein-free, with BSA and with trypsin. Evidently, $T_{\text {LLS }}\left({ }^{2} \mathrm{H}\right)$ 291 and $T_{1}\left({ }^{13} \mathrm{C}\right)$ vary significantly in all three cases. Even in the 292 absence of any target proteins, variations of $12<T_{1}\left({ }^{13} \mathrm{C}\right)<18$ $293 \mathrm{~S}$ were observed. Although not observed, slight fluctuations in 294 sample temperature after dissolution and mixing cannot be 295 ruled out completely. However, the considerable variations of $296 T_{\mathrm{LLS}}\left({ }^{2} \mathrm{H}\right)$ and $T_{1}\left({ }^{13} \mathrm{C}\right)$ cannot be attributed to variations in 297 correlation times $\tau_{\mathrm{c}}$ of overall rotational diffusion as a 298 consequence of temperature fluctuations. Furthermore, control 299 experiments without radicals indicated that $T_{1}\left({ }^{13} \mathrm{C}\right)$ of DMSO$300 d_{6}$ is not significantly affected by the presence of the target 301 proteins: $T_{1}\left({ }^{13} \mathrm{C}\right)=33.7 \pm 5.2 \mathrm{~s}$ in the absence of any protein 302 and $T_{1}\left({ }^{13} \mathrm{C}\right)=30.9 \pm 4.7 \mathrm{~s}$ in the presence of BSA. Thus, the 303 wide range $10<T_{1}\left({ }^{13} \mathrm{C}\right)<30 \mathrm{~s}$ observed in Figure 6 can only 304 be ascribed to varying concentrations of paramagnetic species. 305 As shown in Figure S5 in the SI, the value of $T_{1}\left({ }^{13} \mathrm{C}\right)$ can 306 provide a measure of the TEMPOL concentration. Variations 307 of $12<T_{1}\left({ }^{13} \mathrm{C}\right)<30$ s correspond to $0.08>R_{1}\left({ }^{13} \mathrm{C}\right)>0.033$ $308 \mathrm{~s}^{-1}$ that translates into an estimation of the TEMPOL 309 concentrations in a range between ca. 1 and $4 \mathrm{mM}$ (see 310 Figure S5 in the SI). At least three methods could help to 311 overcome the problem of variable radical concentrations: filtration of radicals attached to surfaces of porous solids, ${ }^{29} 312$ scavenging of the radicals by chemical reduction, ${ }^{30}$ or a rapid 313 transfer of hyperpolarized solid samples combined with 314 subsequent dissolution near the NMR spectrometer. ${ }^{31} \quad 315$

The parameters $T_{\mathrm{LLS}}\left({ }^{2} \mathrm{H}\right)$ and $T_{1}\left({ }^{13} \mathrm{C}\right)$ in Figure 6 are 316 roughly linearly correlated for the three mixtures (protein-free, 317 BSA, or trypsin), which is in line with the linear dependence of 318 slow relaxation rates on radical concentrations ("relaxivity"). 319 The slopes of $T_{\mathrm{LLS}}\left({ }^{2} \mathrm{H}\right)$ vs $T_{1}\left({ }^{13} \mathrm{C}\right)$ are distinct for the three 320 cases. Indeed, the slope is largest in the absence of any protein, 321 corroborating our finding that $T_{\mathrm{LLS}}\left({ }^{2} \mathrm{H}\right)$ tends to be longer in 322 the absence of proteins.

Clearly, improved control over the radical concentration 324 would greatly facilitate the quantitative use of D-DNP for 325 interaction studies. To improve the reliability of D-DNP, 326 $T_{\text {LLS }}\left({ }^{2} \mathrm{H}\right)$ times should ideally be compared with similar 327 TEMPOL concentrations (as in Figure 5) or by considering 328 characteristic slopes $\alpha=\Delta T_{\mathrm{LLS}}\left({ }^{2} \mathrm{H}\right) / \Delta T_{1}\left({ }^{13} \mathrm{C}\right)$ in correlation 329 plots such as in Figure 6 or, if possible, by taking precautions to 330 remove the radicals.

In conclusion, the lifetime $T_{\text {LLS }}\left({ }^{2} \mathrm{H}\right)$ of the A/E imbalance in 332 deuterated methyl groups is longest in the absence of any 333 protein as the methyl group rotation is not perturbed. The 334 interactions between DMSO- $d_{6}$ and BSA or trypsin slow down 335 the methyl group rotation, leading to a shortened $T_{\mathrm{LLS}}\left({ }^{2} \mathrm{H}\right) .336$ Hence, the lifetime $T_{\mathrm{LLS}}\left({ }^{2} \mathrm{H}\right)$ is a good indicator of weak 337 interactions of DMSO- $d_{6}$ with macromolecules such as 338 proteins. The method can be generalized to other ligands 339 with deuterated methyl groups and may offer a novel approach 340 to study weak affinities of deuterated drugs where other 341 techniques might lack sensitivity. Furthermore, it can provide 342 information about a complex's morphology as the internal 343 methyl group dynamics will only be perturbed if the group 344 takes part in the formation of the interaction interface. 345

LLS in deuterated methyl groups represent a complementary 346 approach to other, more or less established, drug-screening 347 techniques such as STD spectroscopy, ${ }^{32}$ Water-LOGSY, ${ }^{11} 348$ contrast of transverse relaxation rates $R_{2}$ or $R_{1 \rho}$ due to 349 binding, ${ }^{6,33}$ contrast of relaxation rates $R_{\mathrm{LLC}}$ of long-lived 350 coherences, ${ }^{34}$ or contrast of relaxation rates $R_{\text {LLS }}$ of long-lived 351 states. $^{7}$ Such techniques usually rely on the detection of nuclei 352 with high gyromagnetic ratios, such as ${ }^{1} \mathrm{H}$ or ${ }^{19} \mathrm{~F}$, because 353 detection of ${ }^{13} \mathrm{C}$ nuclei in natural abundance often does not 354 provide sufficient sensitivity for ligand titration experiments. 355 Hyperpolarization of ${ }^{13} \mathrm{C}$ nuclei can overcome this hurdle, and 356 the indirect detection via ${ }^{13} \mathrm{C}$ of the lifetimes $T_{\mathrm{LLS}}\left({ }^{2} \mathrm{H}\right)$ in 357 hyperpolarized methyl groups can provide an attractive means 358 to characterize weak interactions.

359

Because the dispersion of chemical shifts is more favorable 360 for ${ }^{13} \mathrm{C}$ than that for ${ }^{1} \mathrm{H}$, the method presented here allows one 361 to study a mixture of ligands in a single experiment, provided 362 that the ${ }^{13} \mathrm{C}$ multiplets of the methyl groups of different ligands 363 do not overlap. It remains to be seen to what extent the 364 methodology presented here will be complementary to ${ }^{1} \mathrm{H}-365$ based STD, Water-LOGSY, and $R_{2}$ or $R_{1 \rho}$ studies at millimolar 366 concentrations.

\section{ASSOCIATED CONTENT}

368

Supporting Information

369

The Supporting Information is available free of charge on the 370 ACS Publications website at DOI: 10.1021/acs.jp- 371 clett.9b00149. 


\section{Corresponding Authors}

381 *E-mail: dennis.kurzbach@univie.ac.at.

382 *E-mail: geoffrey.bodenhausen@ens.fr.

3 ORCID ${ }^{\circ}$

384 Thomas Kress: 0000-0001-9133-4310

385 Astrid Walrant: 0000-0003-4054-905X

386 Dennis Kurzbach: 0000-0001-6455-2136

Notes

388 The authors declare no competing financial interest.

\section{ACKNOWLEDGMENTS}

390 The authors are indebted to Dr. Konstantin V. Ivanov 391 (Novosibirsk) for his theoretical insight and to Dr. Mathieu 392 Baudin, Mr. Behdad Aghelnejad, Mr. David Guarin, and Dr. 393 Daniel Abergel for support with experiments. Bruker BioSpin 394 provided a prototype of a D-DNP polarizer. This work was 395 supported by the French CNRS and the ERC (contracts 396 "dilute para water", Grant Agreement 339754, and "HYPRO397 TIN”, Grant Agreement 801936).

\section{DEDICATION}

399 Dedicated to Prof. Hans Dahn on the occasion of his 100th 400 birthday.

\section{$401 \square$ REFERENCES}

402 (1) Di Costanzo, L.; Moulin, M.; Haertlein, M.; Meilleur, F.; 403 Christianson, D. W. Expression, Purification, Assay, and Crystal 404 Structure of Perdeuterated Human Arginase I. Arch. Biochem. Biophys. 405 2007, 465, 82-89.

406 (2) Gant, T. G. Using Deuterium in Drug Discovery: Leaving the 407 Label in the Drug. J. Med. Chem. 2014, 57, 3595-3611.

408 (3) Braman, V.; Graham, P.; Cheng, C.; Turnquist, D.; Harnett, M.; 409 Sabounjian, L. A.; Shipley, J. A Randomized Phase i Evaluation of 410 CTP-499, a Novel Deuterium-Containing Drug Candidate for 411 Diabetic Nephropathy. Clin. Pharmacol. Drug Dev. 2013, 2, 53-66.

412 (4) Uttamsingh, V.; Gallegos, R.; Liu, J. F.; Harbeson, S. L.; Bridson, 413 G. W.; Cheng, C.; Wells, D. S.; Graham, P. B.; Zelle, R.; Tung, R. 414 Altering Metabolic Profiles of Drugs by Precision Deuteration: 415 Reducing Mechanism-Based Inhibition of CYP2D6 by Paroxetine. J. 416 Pharmacol. Exp. Ther. 2015, 354, 43-54.

417 (5) Vuichoud, B.; Milani, J.; Bornet, A.; Melzi, R.; Jannin, S.; 418 Bodenhausen, G. Hyperpolarization of Deuterated Metabolites via 419 Remote Cross-Polarization and Dissolution Dynamic Nuclear Polar420 ization. J. Phys. Chem. B 2014, 118, 1411-1415.

421 (6) Hajduk, P. J.; Olejniczak, E. T.; Fesik, S. W. One-Dimensiona 422 Relaxation- and Diffusion-Edited NMR Methods for Screening 423 Compounds That Bind to Macromolecules. J. Am. Chem. Soc. 1997, 424 119, 12257-12261.

425 (7) Buratto, R.; Bornet, A.; Milani, J.; Mammoli, D.; Vuichoud, B.; 426 Salvi, N.; Singh, M.; Laguerre, A.; Passemard, S.; Gerber-Lemaire, S.; 427 et al. Drug Screening Boosted by Hyperpolarized Long-Lived States in 428 NMR. ChemMedChem 2014, 9, 2509-2515.

429 (8) Mayer, M.; Meyer, B. Group Epitope Mapping by Saturation 430 Transfer Difference NMR To Identify Segments of a Ligand in Direct 431 Contact with a Protein Receptor. J. Am. Chem. Soc. 2001, 123, 61084326117.
(9) Aguirre, C.; Cala, O.; Krimm, I. Overview of Probing Protein- 433 Ligand Interactions Using NMR. Curr. Protoc. Protein Sci. 2015, 2015, 434 17.18.1-17.18.24

(10) Dalvit, C.; Fogliatto, G.; Stewart, A.; Veronesi, M.; Stockman, 436 B. WaterLOGSY as a Method for Primary NMR Screening: Practical 437 Aspects and Range of Applicability. J. Biomol. NMR 2001, 21, 349- 438 359.

(11) Chappuis, Q.; Milani, J.; Vuichoud, B.; Bornet, A.; Gossert, A. 440 D.; Bodenhausen, G.; Jannin, S. Hyperpolarized Water to Study 441 Protein-Ligand Interactions. J. Phys. Chem. Lett. 2015, 6, 1674-1678. 442

(12) Lerche, M. H.; Meier, S.; Jensen, P. R.; Baumann, H.; Petersen, 443 B. O.; Karlsson, M.; Duus, J.; Ardenkjær-Larsen, J. H. Study of 444 Molecular Interactions with ${ }^{13} \mathrm{C}$ DNP-NMR. J. Magn. Reson. 2010, 445 203, 52-56.

(13) Lee, Y.; Zeng, H.; Ruedisser, S.; Gossert, A. D.; Hilty, C. 447 Nuclear Magnetic Resonance of Hyperpolarized Fluorine for 448 Characterization of Protein-Ligand Interactions. J. Am. Chem. Soc. 449 2012, 134, 17448-17451.

(14) Kim, Y.; Liu, M.; Hilty, C. Parallelized Ligand Screening Using 451 Dissolution Dynamic Nuclear Polarization. Anal. Chem. 2016, 88, 452 11178-11183.

(15) Keshari, K. R.; Kurhanewicz, J.; MacDonald, J. M.; Wilson, D. 454 M. Generating Contrast in Hyperpolarized ${ }^{13} \mathrm{C}$ MRI Using Ligand- 455 Receptor Interactions. Analyst 2012, 137, 3427-3429.

(16) Ivanov, K. L.; Kress, T.; Baudin, M.; Guarin, D.; Abergel, D.; 457 Bodenhausen, G.; Kurzbach, D. Relaxation of Long-Lived Modes in 458 NMR of Deuterated Methyl Groups. J. Chem. Phys. 2018, 149, 459 054202 .

(17) Meier, B.; Dumez, J. N.; Stevanato, G.; Hill-Cousins, J. T.; Roy, 461 S. S.; Hakansson, P.; Mamone, S.; Brown, R. C. D.; Pileio, G.; Levitt, 462 M. H. Long-Lived Nuclear Spin States in Methyl Groups and 463 Quantum-Rotor-Induced Polarization. J. Am. Chem. Soc. 2013, 135, 464 18746-18749.

(18) Bornet, A.; Ji, X.; Mammoli, D.; Vuichoud, B.; Milani, J.; 466 Bodenhausen, G.; Jannin, S. Long-Lived States of Magnetically 467 Equivalent Spins Populated by Dissolution-DNP and Revealed by 468 Enzymatic Reactions. Chem. - Eur. J. 2014, 20, 17113-17118. 469

(19) Mammoli, D.; Vuichoud, B.; Bornet, A.; Milani, J.; Dumez, J.- 470 N.; Jannin, S.; Bodenhausen, G. Hyperpolarized Para-Ethanol. J. Phys. 471 Chem. B 2015, 119, 4048-4052.

(20) Kurzbach, D.; Weber, E. M. M.; Jhajharia, A.; Cousin, S. F.; 473 Sadet, A.; Marhabaie, S.; Canet, E.; Birlirakis, N.; Milani, J.; Jannin, S.; 474 Eshchenko, D.; Hassan, A.; Melzi, R.; Luetolf, S.; Sacher, M.; Rossire, 475 M.; Kempf, J.; Lohman, J. A. B.; Weller, M.; Bodenhausen, G.; 476 Abergel, D. Dissolution Dynamic Nuclear Polarization of Deuterated 477 Molecules Enhanced by Cross-Polarization. J. Chem. Phys. 2016, 145, 478 194203.

(21) Jhajharia, A.; Weber, E. M. M.; Kempf, J. G.; Abergel, D.; 480 Bodenhausen, G.; Kurzbach, D. Communication: Dissolution DNP 481 Reveals a Long-Lived Deuterium Spin State Imbalance in Methyl 482 Groups. J. Chem. Phys. 2017, 146, 041101 1-4 .

(22) Buratto, R. Mammoli, D.; Chiarparin, E.; Williams, G.; 484 Bodenhausen, G. Exploring Weak Ligand-Protein Interactions by 485 Long-Lived NMR States: Improved Contrast in Fragment-Based 486 Drug Screening. Angew. Chem., Int. Ed. 2014, 53, 11376-11380. 487

(23) Guarin, D.; Marhabaie, S.; Rosso, A.; Abergel, D.; 488 Bodenhausen, G.; Ivanov, K. L.; Kurzbach, D. Characterizing Thermal 489 Mixing Dynamic Nuclear Polarization via Cross-Talk between Spin 490 Reservoirs. J. Phys. Chem. Lett. 2017, 8, 5531-5536.

(24) Vuichoud, B.; Milani, J.; Chappuis, Q.; Bornet, A.; 492 Bodenhausen, G.; Jannin, S. Measuring Absolute Spin Polarization 493 in Dissolution-DNP by Spin PolarimetrY Magnetic Resonance (SPY- 494 MR) Dedicated to Stefan Schäublin, Alfred Höhener and Richard 495 Ernst for Their Pioneering Work. J. Magn. Reson. 2015, 260, 127- 496 135 .

(25) Bengs, C.; Levitt, M. H. SpinDynamica: Symbolic and 498 Numerical Magnetic Resonance in a Mathematica Environment. 499 Magn. Reson. Chem. 2018, 56, 374-414. 
501 (26) Dumez, J. N.; Håkansson, P.; Mamone, S.; Meier, B.; 502 Stevanato, G.; Hill-Cousins, J. T.; Roy, S. S.; Brown, R. C. D.; 503 Pileio, G.; Levitt, M. H. Theory of Long-Lived Nuclear Spin States in 504 Methyl Groups and Quantum-Rotor Induced Polarisation. J. Chem. 505 Phys. 2015, 142, 044506.

506 (27) Farell, E. M.; Alexandre, G. Bovine Serum Albumin Further 507 Enhances the Effects of Organic Solvents on Increased Yield of 508 Polymerase Chain Reaction of GC-Rich Templates. BMC Res. Notes $5092012,5,257$.

510 (28) Reichenwallner, J.; Hinderberger, D. Using Bound Fatty Acids 511 to Disclose the Functional Structure of Serum Albumin. Biochim. 512 Biophys. Acta, Gen. Subj. 2013, 1830, 5382-5393.

513 (29) Vuichoud, B.; Bornet, A.; de Nanteuil, F.; Milani, J.; Canet, E.; 514 Ji, X.; Miéville, P.; Weber, E.; Kurzbach, D.; Flamm, A.; et al. 515 Filterable Agents for Hyperpolarization of Water, Metabolites, and 516 Proteins. Chem. - Eur. J. 2016, 22, 14696-14700.

517 (30) Miéville, P.; Ahuja, P.; Sarkar, R.; Jannin, S.; Vasos, P.; Gerber518 Lemaire, S.; Mishkovsky, M.; Comment, A.; Gruetter, R.; Ouari, O.; 519 Tordo, P.; Bodenhausen, G. Scavenging Free Radicals to Preserve 520 Enhancement and Extend Relaxation Times in NMR using Dynamic 521 Nuclear Polarization. Angew. Chem., Int. Ed. 2010, 49, 6182-6185.

522 (31) Kouruil, K.; Kouruilová, H.; Levitt, M. H.; Meier, B. Dissolution523 Dynamic Nuclear Polarization with Rapid Transfer of a Polarized 524 Solid. arXiv:1807.00223; 2018.

525 (32) Cala, O.; Guillière, F.; Krimm, I. NMR-Based Analysis of 526 Protein-Ligand Interactions. Anal. Bioanal. Chem. 2014, 406, $943-$ 527956.

528 (33) Kowalewski, J.; Mäler, L. In Nuclear Spin Relaxation in Liquids; 529 Moore, J. H., Spencer, N. D., Eds.; Series in Chemical Physics; Taylor 530 \& Francis, 2006; Vol. 2.

531 (34) Singh, M.; Soni, V. K.; Mishra, R.; Kurur, N. D. Relaxation 532 Editing Using Long-Lived States and Coherences for Analysis of 533 Mixtures. Anal. Chem. 2016, 88, 3004-3008. 